\title{
Productivity, Leaf Nutrient Content and Soil Carbon Stocked in Agroforestry and Traditional Management of Maize (Zea mays L.)
}

\author{
Maria J. A. Bertalot ${ }^{*}$, Iraê A. Guerrini² ${ }^{2}$ Eduardo Mendoza ${ }^{1}$, Mauro S. V. Pinto ${ }^{3}$ \\ ${ }^{1}$ Brazilian Association of Biodynamic Agriculture, Botucatu, Brazil \\ ${ }^{2}$ Department of Natural Resources/Soil Science, FCA, UNESP, Botucatu, SP, Brazil \\ ${ }^{3}$ Embrapa Agroindústria de Alimentos, Rio de Janeiro, Brazil \\ Email: maria@elo.org.br
}

Received 20 December 2013; revised 26 February 2014; accepted 13 March 2014

Copyright (C) 2014 by authors and Scientific Research Publishing Inc.

This work is licensed under the Creative Commons Attribution International License (CC BY). http://creativecommons.org/licenses/by/4.0/

(c) (7) Open Access

\section{Abstract}

The aim of the work was to evaluate the productivity, leaf nutrient content and soil nutrient concentration in maize (Zea mays L.) grown in sequence with black oats (Avena strigosa Schreb.) under Leucaena diversifolia alley cropping agroforestry system (AFS) and traditional management system/sole crop (without trees-TS), after two years of cultivation following a randomized block design. The experiment was carried out in the Brazilian Association of Biodynamic Agriculture, in Botucatu-São Paulo, Brazil. Treatments were: control (C), chemical fertilizer application (F), biomass of $L$. diversifolia alley cropping application (B), biomass of $L$. diversifolia alley cropping + chemical fertilizer application $(B+F)$. In the second year of management it was observed that black oat yield was higher in treatments $B+F$ and $F$ with significant difference in relation to the others treatments in both systems, followed by treatment B. Between systems, only treatment $B$ showed significant difference, with higher yield value corresponding to AFS, reflecting the efficiency of AFS to promote soil fertility. Maize production presented the second year of cultivation an increasing trend in all treatments in both production systems. This result may be due to the cumulative effect of mineralization and maize straw and oats, along the experiment. How productivity was higher in the AFS system, could also be occurring effect of biological nitrogen fixation, water retention and reduction of extreme microclimate through the rows of $L$. diversifolia. Comparing the AFS and TS, it was observed that the concentration of $N$ in leaf tissue was higher in the AFS treatments, probably due to nitrogen fixation performed through the rows of $L$. diversifolia, that is a nitrogen fixing tree species. After two years, carbon stocked in soil show higher values in the treatments biomass + fertilizer and biomass application, in both systems, AFS and TS.

\footnotetext{
"Corresponding author.
}

How to cite this paper: Bertalot, M.J.A., Guerrini, I.A., Mendoza, E. and Pinto, M.S.V. (2014) Productivity, Leaf Nutrient Content and Soil Carbon Stocked in Agroforestry and Traditional Management of Maize (Zea mays L.). American Journal of Plant Sciences, 5, 884-898. http://dx.doi.org/10.4236/ajps.2014.56101 


\section{Keywords}

\section{Alley Cropping; Leucaena diversifolia; Crop Sequence; Green Manure; Soil Nutrient Concentration; Soil Organic Matter; Carbon Stocked in Soil}

\section{Introduction}

Agroforestry systems are agricultural practices with low energy demand, inputs and/or resources, as well as productive and sustainable systems and combine trees and shrubs (especially species of legumes) and food crops and/or forage [1]-[5]. Nair (1985) [6] classified agroforestry systems as agrosilviculture, silvopastoral and agrosilvopastoral.

Alley cropping is a type of agroforestry system consisting of simultaneous combination of trees and/or shrubs, usually nitrogen-fixing species, banded interspersed with annual crops. Trees or shrubs are pruned regularly to use biomass as green manure and/or firewood, with the main objective of improving soil fertility and/or quality fodder. This system also promotes the use of green manure (Kang et al., 1990 [4]; MacDicken and Vergara, 1990 [7]; Bertalot et al., 2004 [8]; Primavesi, 1981 [9]; Schumacher et al., 2003 [10]; Schumacher et al., 2004 [11]). Bertalot et al., 2010 [12]; Bertalot et al., 2008 [13] and Schmitz (2012) [14] reported higher production and economical gains in Leucaena diversifolia alley cropping systems with maize grown in rotation with black oats.

Alley-cropping agroforestry systems let to obtain a productive and sustainable use of land along with diversification of income sources for the small farmer (Kaya and Nair, 2001 [15]; Copijn, 1988 [16]) and have the following characteristics: the practices of cultivation and fallow are held simultaneously; with longer period of cultivation and more intensive use of land; gives a more effective regeneration of soil fertility with more efficient species and reduces the requirements of external inputs. Bertalot (1997) [17] and Bertalot and Mendoza (1998) [18] reported that one of the greatest potentials of alley cropping is its use in sloping areas, where the rows of trees can help reduce erosion by forming natural terraces.

Haggar et al. (1993) [19] used two maize alley cropping treatments, with Erythrina poeppigiana and with Gliricidia sepium, and compared to sole-cropped maize (Zea mays L.) in an 8 yr old experiment at CATIE in Costa Rica. Maize biomass and maize N content, $\mathrm{N}$ released from mulch and residue decomposition, and $\mathrm{N}$ mineralization were all higher in the alley crop than the sole crop.

Marin et al. (2006) [20] and Marin et al. (2007) [21] evaluated biomass productivity of maize, intercropped or not with gliricidia (Gliricidia sepium), and the effect of manure and gliricidia pruning fertilization on maize and spontaneous vegetation biomass productivity. The work was conducted within an area with gliricidia rows, and spaced six meters between each other and one meter between trees. Maize and spontaneous vegetation biomass productivity did not differ between plots fertilized with manure or gliricidia pruning, but these two organic fertilizers led to significantly greater biomass productivity compared to the control plots.

Bertalot et al. (2010) [12] evaluated the productivity of maize in sequence with black oat (Avena strigosa Schreb.) under Leucaena diversifolia alley cropping agroforestry system and traditional management (without trees) in Botucatu, Brazil, and concluded that both systems showed viability from the productive viewpoint and that the biomass use can substitute chemical fertilizer either partially or totally. Siqueira (1999) [22] shown maize in sequence with Raphanus raphanistrum as soil coverage obtaining similar production of biomass as winter crop.

Sharma (2011) [23] studied four ten-year-old land use systems. The systems include: a) agri-horticultural system (Psidium guajaba + Sorghum bicolor); b) agro-forestry system (Acacia auriculiformis + Sorghum bicolor); c) pastoral system (Stylosanthes hamata) and d) arable land system. From the viewpoint of chemical soil quality, agroforestry system proved most in terms of maintaining higher chemical soil quality index compared to other land use systems. Arable land, which is continuously under agriculture, maintained the lowest soil quality index.

The objective of the present study was to evaluate the productivity, leaf nutrient content soil fertility and carbon stocked in soil in an agroforestry system with Leucaena diversifolia and a traditional system (without trees), after two years of cultivation of maize (Zea mays) in rotation with black oats (Avena strigosa).

\section{Material and Methods}

The experiment was carried out from may 2000 to april 2002 at the Brazilian Association of Biodynamic Agri- 
culture (ABD) in Botucatu, São Paulo, Brazil (latitude $22^{\circ} 57^{\prime} \mathrm{S}$, longitude $48^{\circ} 25^{\prime} \mathrm{W}$ ), in the Cerrado area at an altitude of 900 meters above sea level. The local climate is classified as "cfa" (subtropical wet), according to Köppen.

The average annual temperature is $20.5^{\circ} \mathrm{C}$ and average annual rainfall is $1.549 \mathrm{~mm}$, occurring in greater quantities from November to March. The soil of the region is classified as red-yellow latosol (oxisol), medium textured (Jorge, 1986 [24]; Tomé Junior, 1997 [25]).

The experiment was carried out in two different areas, one of them managed under an agroforestry system of alleys of Leucaena diversifolia (AFS) and another area under traditional management system/sole crop (TS). The Leucaena diversifolia has already been planted with success in the Biodynamic Association area since 1995, due to its adaptability to Cerrado soils (Bertalot et al., 2002) [26]. At the time of pruning, the material of L. diversifolia was cut, weighed and dried for chemical analysis of nutritional status according to methodology developed by Malavolta et al. (1997) [27] Figure 1.

The analysis of vegetative material from $L$. diversifolia pruning (leaves and branches) showed the following nutrient content: $31 \mathrm{~g} \cdot \mathrm{kg}^{-1} \mathrm{~N}, 1.4 \mathrm{~g} \cdot \mathrm{kg}^{-1} \mathrm{P}, 15 \mathrm{~g} \cdot \mathrm{kg}^{-1} \mathrm{~K}, 6 \mathrm{~g} \cdot \mathrm{kg}^{-1} \mathrm{Ca}, 2.4 \mathrm{~g} \cdot \mathrm{kg}^{-1} \mathrm{Mg}, 1.6 \mathrm{~g} \cdot \mathrm{kg}^{-1} \mathrm{~S}, 21 \mathrm{mg} \cdot \mathrm{kg}^{-1} \mathrm{~B}$, $31 \mathrm{mg} \cdot \mathrm{kg}^{-1} \mathrm{Cu}, 143 \mathrm{mg} \cdot \mathrm{kg}^{-1} \mathrm{Fe}, 36 \mathrm{mg} \cdot \mathrm{kg}^{-1} \mathrm{Mn}$ and $10 \mathrm{mg} \cdot \mathrm{kg}^{-1} \mathrm{Zn}$ (Bertalot, 2003 [28], Leal et al., 1993 [29]).

Annual crops used to conduct the experiment in both areas were: black oats (Avena strigosa), grown as winter crop and used as green manure, in rotation with maize (Zea mays), grown as summer crop.

Before installing the experiment, soil samples representative of the area were collected at a depth of $0-20 \mathrm{~cm}$, to perform physical analysis by the hydrometer method (Embrapa, 1997) [30] and chemical analysis by the methodology described by Raij (1983) [31] and Raij et al. (2001) [32]. Results are presented in Table 1.

AFS

\begin{tabular}{|c|c|c|c|c|}
\hline L. diversifolia & L. diversifolia & L. diversifolia & L. diversifolia & $1 \mathrm{~m}$ \\
\hline Crop T1 & Crop T2 & Crop T3 & Crop T4 & $\uparrow$ \\
\hline L. diversifolia & L. diversifolia & L. diversifolia & L. diversifolia & $1 \mathrm{~m}$ \\
\hline \multicolumn{5}{|c|}{ Traditional systems } \\
\hline Crop T1 & Crop T2 & Crop T3 & Crop T4 & $\underset{5 \mathrm{~m}}{1}$ \\
\hline
\end{tabular}

Figure 1. Design of agroforestry and traditional systems.

Table 1. Chemical and physical soil analysis before the installation of the trial ( 0 - $20 \mathrm{~cm}$ depth) in agroforestry system (AFS) and traditional system (TS).

\begin{tabular}{|c|c|c|c|c|c|c|c|c|c|c|c|c|c|c|c|c|}
\hline \multicolumn{17}{|c|}{ Chemical analysis } \\
\hline & $\mathrm{pH}$ & $\mathrm{OM}$ & $\mathrm{C}$ & $P$ resin & $\mathrm{Al}^{3+}$ & $\mathrm{H}+\mathrm{Al}$ & $\mathrm{K}$ & $\mathrm{Ca}$ & $\mathrm{Mg}$ & CEC & BS & $\mathrm{B}$ & $\mathrm{Cu}$ & $\mathrm{Fe}$ & $\mathrm{Mn}$ & $\mathrm{Zn}$ \\
\hline & $\mathrm{CaCl}_{2}$ & \multicolumn{2}{|c|}{$\mathrm{g} \cdot \mathrm{dm}^{-3}$} & $\mathrm{mg} \cdot \mathrm{dm}^{-3}$ & \multicolumn{6}{|c|}{ mmolc $\cdot \mathrm{dm}^{-3}$} & $\%$ & \multicolumn{5}{|c|}{$\mathrm{mg} \cdot \mathrm{dm}^{-3}$} \\
\hline AFS & 5.2 & 17 & 9.88 & 17 & 0.6 & 20 & 0.4 & 19 & 9.4 & 49.2 & 59.4 & 0.14 & 088 & 29 & 0.9 & 0.44 \\
\hline TS & 5.2 & 16 & 9.30 & 14 & 0.6 & 19 & 0.3 & 17 & 9.0 & 45.6 & 57.6 & 0.11 & 1.1 & 32 & 0.5 & 0.26 \\
\hline
\end{tabular}




\section{Treatments}

Treatments for both systems were:

1) Control (C);

2) Application of chemical fertilizer $(F)$;

3) Application of prunings (biomass) of Leucaena diversifolia (B);

4) Application of prunings (biomass) of Leucaena diversifolia + chemical fertilizer (B + F).

In all treatments, including Control, crop residues were kept over the soil, beginning with black oat straw and maize stover in the first year of cultivation. The experimental design consisted of randomized blocks with four treatments and five replicates in both cropping systems. Each plot occupied an area of $20 \mathrm{~m}^{2}$ (5 m wide $\times 4 \mathrm{~m}$ long), and $1 \mathrm{~m}$ border between plots.

Analysis of variance was performed by Estat D Program, developed by specialists of the University of the São Paulo State-UNESP, Jaboticabal—SP, Brazil. Tukey test was used to compare mean values and a joint analysis to compare systems was carried out, in accordance to Banzatto and Kronka, 1995 [33].

“Tables 2 to 10 have statistical analysis in accordance to Banzatto and Kronka (1995) [33]”.

Soil Carbon Stock at 0 - 20 cm depth

Chemical and physical analysis of soil after two years of management were reported by Bertalot (2003) and Bertalot et al. (2013) [34].

Table 2. Leaf nutrient concentration in black oats tissue in AFS and TS—first year of management.

\section{AFS}

\section{Nutrient concentration}

\begin{tabular}{|c|c|c|c|c|c|c|c|c|c|c|c|}
\hline \multicolumn{7}{|c|}{$\mathrm{g} \cdot \mathrm{kg}^{-1}$} & \multicolumn{5}{|c|}{$\mathrm{mg} \cdot \mathrm{kg}^{-1}$} \\
\hline Treatment & $\mathrm{N}$ & $\mathrm{P}$ & K & $\mathrm{Ca}$ & $\mathrm{Mg}$ & S & B & $\mathrm{Cu}$ & $\mathrm{Fe}$ & Mn & $\mathrm{Zn}$ \\
\hline $\mathrm{C}$ & 15.0 & 1.82 & $10.4 \mathrm{~b}$ & 2.20 & 1.68 & $1.04 \mathrm{~b}$ & 10.8 & $8.20 \mathrm{~b}$ & $78.00 \mathrm{c}$ & 64.40 & $17.00 \mathrm{~b}$ \\
\hline F & 17.8 & 2.00 & $12.8 \mathrm{a}$ & 2.20 & 1.84 & $1.72 \mathrm{a}$ & 12.0 & $11.2 \mathrm{a}$ & $114.40 \mathrm{a}$ & 73.00 & $20.20 \mathrm{ab}$ \\
\hline B & 15.0 & 2.04 & $13.2 \mathrm{a}$ & 2.20 & 1.78 & $1.22 \mathrm{~b}$ & 10.6 & $10.2 \mathrm{ab}$ & 86.20 c & 74.20 & $20.40 \mathrm{ab}$ \\
\hline$B+F$ & 16.0 & 2.08 & $14.0 \mathrm{a}$ & 2.80 & 1.98 & $1.68 \mathrm{a}$ & 11.4 & $10.6 \mathrm{a}$ & $100.20 \mathrm{~b}$ & 74.60 & $24.40 \mathrm{a}$ \\
\hline $\mathrm{F}$ & NS & NS & $* *$ & NS & NS & ** & NS & * & ** & NS & ** \\
\hline LSD & 4.13 & 0.69 & 2.06 & 0.84 & 0.31 & 0.38 & 2.85 & 2.34 & 10.39 & 18.24 & 5.06 \\
\hline CV \% & 13.80 & 18.60 & 8.72 & 19.03 & 9.10 & 14.25 & 15.04 & 12.89 & 5.84 & 13.58 & 13.15 \\
\hline
\end{tabular}

Nutrient concentration

\begin{tabular}{cccccccccccc} 
& \multicolumn{9}{c}{$\mathrm{g}^{\mathrm{kg}} \mathrm{kg}^{-1}$} \\
Treatment & $\mathrm{N}$ & $\mathrm{P}$ & $\mathrm{K}$ & $\mathrm{Ca}$ & $\mathrm{Mg}$ & $\mathrm{S}$ & $\mathrm{B}$ & $\mathrm{Cu}$ & $\mathrm{Fe}$ & $\mathrm{Mn}$ & $\mathrm{Zn}$ \\
$\mathrm{C}$ & $10.8 \mathrm{~b}$ & $1.5 \mathrm{~b}$ & $11.6 \mathrm{c}$ & 1.8 & $1.28 \mathrm{c}$ & 1.22 & $13.4 \mathrm{~b}$ & 7.6 & $79.8 \mathrm{c}$ & $55.8 \mathrm{~b}$ & $23.8 \mathrm{~b}$ \\
$\mathrm{~F}$ & $12.4 \mathrm{a}$ & $1.9 \mathrm{a}$ & $17.6 \mathrm{ab}$ & 1.8 & $1.34 \mathrm{bc}$ & 1.28 & $18.0 \mathrm{a}$ & 8.4 & $83.6 \mathrm{c}$ & $66.2 \mathrm{a}$ & $29.4 \mathrm{a}$ \\
$\mathrm{B}$ & $12.4 \mathrm{a}$ & $1.8 \mathrm{a}$ & $16.8 \mathrm{~b}$ & 2.2 & $1.48 \mathrm{ab}$ & 1.32 & $18.6 \mathrm{a}$ & 9.0 & $103.8 \mathrm{a}$ & $68.0 \mathrm{a}$ & $26.2 \mathrm{ab}$ \\
$\mathrm{B}+\mathrm{F}$ & $12.6 \mathrm{a}$ & $1.9 \mathrm{a}$ & $19.4 \mathrm{a}$ & 2.0 & $1.54 \mathrm{a}$ & 1.38 & $19.4 \mathrm{a}$ & 8.6 & $94.0 \mathrm{~b}$ & $67.2 \mathrm{a}$ & $28.8 \mathrm{a}$ \\
$\mathrm{F}$ & $*$ & $* *$ & $* *$ & $\mathrm{NS}$ & $* *$ & $\mathrm{NS}$ & $* *$ & $\mathrm{NS}$ & $* *$ & $* *$ & $* *$ \\
$\mathrm{LSD}$ & 1.60 & 0.23 & 2.43 & 0.59 & 0.15 & 0.17 & 2.98 & 1.61 & 5.15 & 9.49 & 4.35 \\
$\mathrm{CV} \%$ & 7.07 & 6.9 & 7.9 & 16.2 & 5.68 & 7.26 & 9.16 & 10.19 & 3.03 & 7.86 & 8.56 \\
\hline
\end{tabular}

** and ${ }^{*}$ significant at $1 \%$ and $5 \%$, respectively, by $\mathrm{F}$ test. Means followed by the same letter do not differ among themselves, by Tukey Test (P > 0.05). Source Bertalot (2003). 
Table 3. Nutrient content in dry matter of black oats—-first year of management.

\begin{tabular}{|c|c|c|c|c|c|c|c|c|c|c|c|}
\hline \multicolumn{12}{|c|}{ AFS } \\
\hline \multicolumn{12}{|c|}{ Nutrient content $\left(\mathrm{kg} \cdot \mathrm{ha}^{-1}\right)$} \\
\hline Treatment & $\mathrm{N}$ & $\mathrm{P}$ & $\mathrm{K}$ & $\mathrm{Ca}$ & $\mathrm{Mg}$ & S & B & $\mathrm{Cu}$ & $\mathrm{Fe}$ & Mn & $\mathrm{Zn}$ \\
\hline $\mathrm{C}$ & 89.62 b & 10.56 c & $61.72 \mathrm{~d}$ & $13.34 \mathrm{~b}$ & $10.26 \mathrm{~d}$ & $6.29 \mathrm{~d}$ & $0.66 \mathrm{~b}$ & $0.05 \mathrm{~b}$ & $0.48 \mathrm{~d}$ & $0.38 \mathrm{~d}$ & $0.10 \mathrm{~d}$ \\
\hline $\mathrm{F}$ & 130.17 a & $14.34 \mathrm{ab}$ & $94.06 \mathrm{~b}$ & $16.19 \mathrm{~b}$ & $13.36 \mathrm{~b}$ & $12.41 \mathrm{~b}$ & $0.88 \mathrm{a}$ & $0.08 \mathrm{a}$ & $0.84 \mathrm{a}$ & $0.54 \mathrm{~b}$ & $0.15 \mathrm{~b}$ \\
\hline B & $93.09 \mathrm{~b}$ & 12.41 bc & 81.78 c & $13.53 \mathrm{~b}$ & $11.36 \mathrm{c}$ & $7.35 \mathrm{c}$ & $0.66 \mathrm{~b}$ & $0.06 \mathrm{~b}$ & $0.53 \mathrm{c}$ & $0.46 \mathrm{c}$ & $0.13 \mathrm{c}$ \\
\hline$B+F$ & 131.18 a & 16.36 a & $114.52 \mathrm{a}$ & 22.09 a & $16.53 \mathrm{a}$ & $13.29 \mathrm{a}$ & $0.91 \mathrm{a}$ & $0.08 \mathrm{a}$ & $0.81 \mathrm{~b}$ & $0.61 \mathrm{a}$ & $0.20 \mathrm{a}$ \\
\hline $\mathrm{F}$ & ** & ** & ** & ** & ** & ** & ** & ** & ** & ** & ** \\
\hline LSD & 5.42 & 2.07 & 4.57 & 3.31 & 0.772 & 0.40 & 0.04 & 0.01 & 0.01 & 0.005 & 0.011 \\
\hline CV \% & 2.60 & 8.23 & 23.76 & 10.81 & 2.99 & 2.18 & 2.59 & 12.16 & 1.05 & 0.55 & 4.01 \\
\hline \multicolumn{12}{|c|}{ TS } \\
\hline \multicolumn{12}{|c|}{ Nutrient content $\left(\mathrm{kg} \cdot \mathrm{ha}^{-1}\right)$} \\
\hline Treatment & $\mathrm{N}$ & $\mathrm{P}$ & $\mathrm{K}$ & $\mathrm{Ca}$ & $\mathrm{Mg}$ & S & B & $\mathrm{Cu}$ & $\mathrm{Fe}$ & Mn & $\mathrm{Zn}$ \\
\hline $\mathrm{C}$ & 18.91 c & $2.29 \mathrm{c}$ & $19.69 \mathrm{~d}$ & $3.40 \mathrm{~d}$ & $2.77 \mathrm{c}$ & $2.69 \mathrm{~d}$ & $0.02 \mathrm{c}$ & $0.016 \mathrm{c}$ & $0.13 \mathrm{~d}$ & $0.09 \mathrm{c}$ & $0.04 \mathrm{c}$ \\
\hline $\mathrm{F}$ & 83.55 a & 12.44 a & $118.86 \mathrm{~b}$ & $12.37 \mathrm{~b}$ & 9.32 a & $8.46 \mathrm{~b}$ & $0.12 \mathrm{a}$ & $0.050 \mathrm{a}$ & $0.56 \mathrm{~b}$ & $0.44 \mathrm{a}$ & $0.19 \mathrm{a}$ \\
\hline B & $50.42 \mathrm{~b}$ & $7.31 \mathrm{~b}$ & $68.41 \mathrm{c}$ & $8.47 \mathrm{c}$ & $6.42 \mathrm{~b}$ & $5.62 \mathrm{c}$ & $0.07 \mathrm{~b}$ & $0.038 \mathrm{~b}$ & $0.43 \mathrm{c}$ & $0.28 \mathrm{~b}$ & $0.11 \mathrm{~b}$ \\
\hline $\mathrm{B}+\mathrm{F}$ & 84.27 a & $12.50 \mathrm{a}$ & 132.09 a & $13.50 \mathrm{a}$ & $6.42 \mathrm{~b}$ & $9.42 \mathrm{a}$ & $0.13 \mathrm{a}$ & $0.062 \mathrm{a}$ & $0.64 \mathrm{a}$ & $0.45 \mathrm{a}$ & $0.20 \mathrm{a}$ \\
\hline $\mathrm{F}$ & ** & $* *$ & $* *$ & $* *$ & $* *$ & ** & $* *$ & $* *$ & $* *$ & ** & ** \\
\hline LSD & 1.39 & 0.71 & 1.68 & 0.52 & 0.88 & 0.72 & 0.018 & 0.01 & 0.016 & 0.02 & 0.014 \\
\hline CV \% & 1.25 & 4.40 & 1.05 & 2.95 & 6.48 & 5.88 & 11.60 & 12.71 & 1.96 & 3.20 & 5.69 \\
\hline
\end{tabular}

The determination of organic carbon and organic matter was performed by oxidation method of Walkley \& Black, used by Luske and van der Kamp (2009) [35] and described by Cantarella, Quaggio and Raij (2001) [36]. With the results was obtained the amount of carbon stocked in the soil using the following formula:

\section{Carbon stock in the soil $=$ sampling depth $\mathrm{x}$ carbon content $\mathrm{x}$ bulk density \\ Sowing of black oats.}

Black oats was planted at a spacing of $20 \mathrm{~cm}$ between rows. According to soil analysis (Table 1), it was not necessary to apply limestone because the base saturation was above $50 \%$. Chemical fertilizer was applied at seeding expecting a grain yield of $2-3 \mathrm{t} \cdot \mathrm{ha}^{-1}$ as recommended by Camargo et al. (1997), and was as follows: $300 \mathrm{~kg} \cdot \mathrm{ha}^{-1}$ of $04-20-20+0.1 \%$ B plus $18 \mathrm{~kg} \cdot \mathrm{ha}^{-1}$ of $\mathrm{N}$ in treatments with application of fertilizer (2) and biomass + fertilizer (4), as related by Bertalot (2003) [28].

The chemical cover fertilization consisted of $40 \mathrm{~kg} \cdot \mathrm{N} \cdot \mathrm{ha}^{-1}$ in both years of planting. For treatments with application of biomass of $L$. diversifolia (3), $7.5 \mathrm{t}^{\mathrm{h}} \mathrm{ha}^{-1}$ of dry matter of $L$. diversifolia were added, in accordance with Copijn (1988) [16]. Black oats were cut down at soft grain/dough stage, 120 days after seeding, as suggested by Derpsch and Calegari (1992) [37], and the cut straw was left as ground cover in all plots in both systems.

\section{Maize sowing.}

Maize was seeded with $0.90 \mathrm{~m}$ spacing between rows, five plants per meter. Planting density was 55,000 plants per hectare. Soil samples of treatments Biomass + fertilizer (4) and Fertilizer (2) were taken from in both systems. Limestone was applied to raise the base saturation to $70 \%\left(0.43 \mathrm{t}^{-h a^{-1}}\right)$, in accordance with Raij et al. (2001) [32] and Levien (1999) [38]. 
Table 4. Mean productivity of grains of Maize both the systems - first year of management $\left(\mathrm{t} \cdot \mathrm{ha}^{-1}\right)$.

\begin{tabular}{ccc}
\hline Treatment & \multicolumn{2}{c}{${\text { Maize first year Mean Productivity }\left(\mathbf{t} \cdot \mathbf{h a}^{-1}\right)}^{-1}$} \\
Control & AFS & TS \\
Fertilizer & $6.10 \mathrm{bA}$ & $4.38 \mathrm{cB}$ \\
Biomass & $8.79 \mathrm{aA}$ & $6.68 \mathrm{abB}$ \\
Biomass + fertilizer & $8.50 \mathrm{aA}$ & $5.49 \mathrm{bcB}$ \\
F & $8.68 \mathrm{aA}$ & $7.62 \mathrm{aA}$ \\
LSD in the columns & $* * * *$ & 1.72 \\
CV & 1.72 & 15.20 \\
\hline
\end{tabular}

${ }^{* *}$ and ${ }^{*}$ significant at $1 \%$ and $5 \%$, respectively, by $\mathrm{F}$ test. Lowercase letters in the columns, comparison between treatments for each system. Capital letter in the lines comparison of systems for each treatment Means followed by the same letter do not differ among themselves, by Tukey Test (P $>0.05$ ). Source Bertalot (2003).

\section{Table 5. Leaf nutrient concentration in corn leaf tissue in AFS and TS-first year of management.}

\section{AFS}

Nutrient concentration

\begin{tabular}{|c|c|c|c|c|c|c|c|c|c|c|c|}
\hline \multicolumn{7}{|c|}{$\mathrm{g} \cdot \mathrm{kg}^{-1}$} & \multicolumn{5}{|c|}{$\mathrm{mg} \cdot \mathrm{kg}^{-1}$} \\
\hline Treatment & $\mathrm{N}$ & $\mathrm{P}$ & K & $\mathrm{Ca}$ & $\mathrm{Mg}$ & $\mathrm{S}$ & B & $\mathrm{Cu}$ & $\mathrm{Fe}$ & $\mathrm{Mn}$ & $\mathrm{Zn}$ \\
\hline C & $24.80 \mathrm{~b}$ & 2.72 & $13.40 \mathrm{~b}$ & 3.40 & 4.06 & 1.76 & 13.20 & $5.40 \mathrm{~b}$ & 116.00 & 27.80 & $3.00 \mathrm{~b}$ \\
\hline $\mathrm{F}$ & $26.60 \mathrm{ab}$ & 2.82 & $15.00 \mathrm{a}$ & 4.20 & 4.76 & 1.84 & 14.20 & $8.80 \mathrm{ab}$ & 131.40 & 36.20 & $4.80 \mathrm{~b}$ \\
\hline B & $26.20 \mathrm{ab}$ & 2.88 & 14.80 a & 3.80 & 4.64 & 1.88 & 14.20 & $9.80 \mathrm{a}$ & 125.80 & 31.20 & $3.00 \mathrm{~b}$ \\
\hline $\mathrm{B}+\mathrm{F}$ & 27.80 a & 3.04 & $16.00 \mathrm{a}$ & 4.00 & 4.14 & 2.16 & 14.40 & $9.80 \mathrm{a}$ & 129.20 & 37.60 & $8.80 \mathrm{a}$ \\
\hline F & $* *$ & NS & $* *$ & NS & NS & NS & NS & $*$ & NS & NS & $* *$ \\
\hline LSD & 2.01 & 0.48 & 1.30 & 1.01 & 1.35 & 0.55 & 3.72 & 4.23 & 19.51 & 10.50 & 2.32 \\
\hline CV \% & 4.07 & 8.92 & 4.70 & 14.03 & 16.39 & 15.35 & 14.15 & 26.66 & 8.27 & 16.84 & 25.20 \\
\hline \multicolumn{12}{|c|}{ TS } \\
\hline \multicolumn{12}{|c|}{ Nutrient concentration } \\
\hline & & & $\mathrm{g} \cdot \mathrm{kg}^{-1}$ & & & & \multicolumn{5}{|c|}{$\mathrm{mg} \cdot \mathrm{kg}^{-1}$} \\
\hline Treatment & $\mathrm{N}$ & $\mathrm{P}$ & $\mathrm{K}$ & $\mathrm{Ca}$ & $\mathrm{Mg}$ & $\mathrm{S}$ & B & $\mathrm{Cu}$ & $\mathrm{Fe}$ & $\mathrm{Mn}$ & $\mathrm{Zn}$ \\
\hline $\mathrm{C}$ & $16.80 \mathrm{c}$ & $1.56 \mathrm{c}$ & $12.80 \mathrm{~b}$ & 2.60 & $2.80 \mathrm{~b}$ & $1.02 \mathrm{c}$ & $11.00 \mathrm{~b}$ & $6.00 \mathrm{c}$ & $65.40 \mathrm{~d}$ & $12.40 \mathrm{c}$ & $18.60 \mathrm{c}$ \\
\hline $\mathrm{F}$ & $21.00 \mathrm{~b}$ & $1.90 \mathrm{~b}$ & $14.00 \mathrm{ab}$ & 3.20 & $3.38 \mathrm{a}$ & $1.26 \mathrm{~b}$ & $13.40 \mathrm{a}$ & $9.80 \mathrm{ab}$ & $74.20 \mathrm{~b}$ & $18.40 \mathrm{~b}$ & $22.00 \mathrm{~b}$ \\
\hline B & 18.60 c & 1.68 bc & $14.00 \mathrm{ab}$ & 2.80 & $2.90 \mathrm{~b}$ & 1.14 bc & $12.80 \mathrm{ab}$ & $8.40 \mathrm{~b}$ & $70.20 \mathrm{c}$ & $17.00 \mathrm{~b}$ & $21.80 \mathrm{~b}$ \\
\hline $\mathrm{B}+\mathrm{F}$ & 26.20 a & $2.30 \mathrm{a}$ & $15.00 \mathrm{a}$ & 3.40 & $3.50 \mathrm{a}$ & $1.60 \mathrm{a}$ & $13.80 \mathrm{a}$ & $11.20 \mathrm{a}$ & 80.00 a & $21.20 \mathrm{a}$ & $25.60 \mathrm{a}$ \\
\hline $\mathrm{F}$ & $* *$ & $* *$ & $*$ & NS & $* *$ & $* *$ & $* *$ & $* *$ & $* *$ & $* *$ & $* *$ \\
\hline LSD & 2.34 & 0.32 & 2.14 & 0.86 & 0.27 & 0.18 & 1.82 & 1.58 & 3.87 & 2.28 & 2.21 \\
\hline $\mathrm{CV} \%$ & 6.27 & 9.21 & 8.17 & 15.21 & 4.55 & 7.80 & 7.61 & 9.51 & 2.85 & 7.04 & 5.36 \\
\hline
\end{tabular}

** and ${ }^{*}$ significant at $1 \%$ and $5 \%$, respectively, by $\mathrm{F}$ test. Means followed by the same letter do not differ among themselves, by Tukey Test (P > 0.05). Source Bertalot (2003). 
Table 6. Leaf nutrient concentration in black oats in AFS and TS-second year of management.

\section{AFS}

Nutrient concentration

\begin{tabular}{|c|c|c|c|c|c|c|c|c|c|c|c|}
\hline \multicolumn{7}{|c|}{$\mathrm{g} \cdot \mathrm{kg}^{-1}$} & \multicolumn{5}{|c|}{$\mathrm{mg} \cdot \mathrm{kg}^{-1}$} \\
\hline Treatment & $\mathrm{N}$ & $\mathrm{P}$ & $\mathrm{K}$ & $\mathrm{Ca}$ & $\mathrm{Mg}$ & S & B & $\mathrm{Cu}$ & $\mathrm{Fe}$ & $\mathrm{Mn}$ & $\mathrm{Zn}$ \\
\hline $\mathrm{C}$ & $14.40 \mathrm{~b}$ & 2.08 & $9.20 \mathrm{c}$ & 2.20 & 1.38 c & $1.18 \mathrm{~b}$ & 5.00 & 3.40 & $72.60 \mathrm{c}$ & $65.40 \mathrm{c}$ & $19.60 \mathrm{~b}$ \\
\hline $\mathrm{F}$ & $16.00 \mathrm{ab}$ & 2.12 & $12.20 \mathrm{~b}$ & 2.40 & $1.55 \mathrm{~b}$ & $1.28 \mathrm{ab}$ & 5.20 & 3.60 & $78.00 \mathrm{ab}$ & 69.80 bc & $21.40 \mathrm{~b}$ \\
\hline B & $16.00 \mathrm{ab}$ & 2.18 & $13.40 \mathrm{ab}$ & 2.40 & $1.67 \mathrm{ab}$ & $1.26 \mathrm{~b}$ & 5.00 & 4.00 & 74.40 bc & $74.40 \mathrm{ab}$ & $21.60 \mathrm{~b}$ \\
\hline$B+F$ & $16.20 \mathrm{a}$ & 2.22 & $14.20 \mathrm{a}$ & 3.00 & $1.89 \mathrm{a}$ & $1.42 \mathrm{a}$ & 5.40 & 4.20 & 80.20 a & 76.60 a & $25.00 \mathrm{a}$ \\
\hline $\mathrm{F}$ & $*$ & NS & $* *$ & NS & $* *$ & $* *$ & NS & NS & $* *$ & $* *$ & $* *$ \\
\hline LSD & 1.69 & 0.22 & 1.54 & 0.89 & 0.14 & 0.15 & 1.81 & 1.15 & 5.07 & 4.91 & 2.50 \\
\hline $\mathrm{CV} \%$ & 5.74 & 5.40 & 6.71 & 18.97 & 4.63 & 6.07 & 18.76 & 16.12 & 3.54 & 3.66 & 6.08 \\
\hline
\end{tabular}

TS

Nutrient concentration

\begin{tabular}{|c|c|c|c|c|c|c|c|c|c|c|c|}
\hline \multicolumn{7}{|c|}{$\mathrm{g} \cdot \mathrm{kg}^{-1}$} & \multicolumn{5}{|c|}{$\mathrm{mg} \cdot \mathrm{kg}^{-1}$} \\
\hline Treatment & $\mathrm{N}$ & $\mathrm{P}$ & K & $\mathrm{Ca}$ & $\mathrm{Mg}$ & $\mathrm{S}$ & B & $\mathrm{Cu}$ & $\mathrm{Fe}$ & $\mathrm{Mn}$ & $\mathrm{Zn}$ \\
\hline $\mathrm{C}$ & 13.8 & 1.90 & $9.40 \mathrm{c}$ & 2.20 & 1.32 & $1.02 \mathrm{~b}$ & 4.20 & 2.80 & $66.40 \mathrm{~b}$ & $52.40 \mathrm{c}$ & 21.40 \\
\hline F & 14.0 & 1.92 & $11.60 \mathrm{~b}$ & 2.60 & 1.34 & $1.30 \mathrm{a}$ & 4.40 & 3.60 & $75.60 \mathrm{a}$ & $66.60 \mathrm{~b}$ & 21.40 \\
\hline B & 13.8 & 1.92 & $12.60 \mathrm{ab}$ & 2.60 & 1.42 & $1.18 \mathrm{a}$ & 4.40 & 3.80 & $71.20 \mathrm{ab}$ & $67.20 \mathrm{ab}$ & 22.00 \\
\hline$B+F$ & 15.2 & 1.98 & 13.80 a & 2.60 & 1.44 & $1.22 \mathrm{a}$ & 4.80 & 4.00 & $75.00 \mathrm{a}$ & 72.40 a & 22.40 \\
\hline $\mathrm{F}$ & NS & NS & $* *$ & NS & NS & $* *$ & NS & NS & $* *$ & $* *$ & NS \\
\hline LSD & 1.59 & 0.201 & 1.49 & 1.07 & 0.14 & 0.13 & 1.12 & 1.39 & 7.16 & 5.27 & 2.70 \\
\hline $\mathrm{CV} \%$ & 6.00 & 5.54 & 6.72 & 22.80 & 5.65 & 5.84 & 13.45 & 20.89 & 5.29 & 4.34 & 6.61 \\
\hline
\end{tabular}

** and ${ }^{*}$ significant at $1 \%$ and $5 \%$, respectively, by F test. Means followed by the same letter do not differ among themselves, by Tukey Test (P > 0.05).

Chemical fertilization at seeding for an expected productivity of 4 - $6 \mathrm{t}^{\mathrm{h}} \mathrm{ha}^{-1}$ of grain, $300 \mathrm{~kg} \cdot \mathrm{ha}^{-1}$ of $04-20-20$ commercial chemical fertilizer $+0.1 \% \mathrm{~B}$, plus $8 \mathrm{~kg}$ of $\mathrm{N}$ were applied.

For chemical cover fertilization $40 \mathrm{~kg} \cdot \mathrm{N} \mathrm{ha}^{-1}$ were applied in the first year of management. In order to raise the base saturation to $70 \%$ in the second year limestone was added to the soil $\left(1 \mathrm{t}^{-h a^{-1}}\right)$. Chemical fertilizer was applied in the seeding. For an expected productivity of $4-6$ tha $^{-1}$ of grain, $250 \mathrm{~kg}$ ha of $08-28-16+0.4 \% \mathrm{Zn}$ were added. Cover fertilization at 40 days was carried out with 40 , in accordance with soil analysis. Green manure was added as $7.5 \mathrm{t} \cdot \mathrm{ha}^{-1}$ of dry matter using prunings of L. diversifolia in Biomass and Biomass + fertilizer treatments in both systems, treatments 3 and 4, respectively (Bertalot, 2003).

No irrigation was applied during the study time. Temperature data and monthly rainfall during the experiment, from May 2000 to April 2002 are shown in Table 11.

\section{Results and Discussion}

Dry matter yield of black oats obtained in the first year of management was 6.00, 7.37, 6.19 and 8.13 tha ${ }^{-1}$ for treatments C, F, B and B + F, respectively in AFS. In TS yield was of 1.70, 6.74, 4.08 and $6.74 \mathrm{t} \cdot \mathrm{ha}^{-1}$, for treatments C, F, B and B + F, respectively, as reported by Bertalot (2003) and Bertalot et al. (2010) Figure 2.

On the AFS, there was no difference in yield between treatments $(B+F)$ and $(F)$. However, the difference between treatments $(\mathrm{B}+\mathrm{F})$ and $(\mathrm{F})$ was $760 \mathrm{~kg} \cdot \mathrm{ha}^{-1}$ of dry matter, provided only by the addition of biomass of 
Table 7. Leaf nutrient content in black oats in AFS and TS—second year of management.

\begin{tabular}{|c|c|c|c|c|c|c|c|c|c|c|c|}
\hline \multicolumn{12}{|c|}{ AFS } \\
\hline \multicolumn{12}{|c|}{ Leaf nutrient content $\left(\mathrm{kg} \cdot \mathrm{ha}^{-1}\right)$} \\
\hline Treatment & $\mathrm{N}$ & $\mathrm{P}$ & $\mathrm{K}$ & $\mathrm{Ca}$ & $\mathrm{Mg}$ & S & B & $\mathrm{Cu}$ & $\mathrm{Fe}$ & $\mathrm{Mn}$ & Zn \\
\hline $\mathrm{C}$ & $85.69 \mathrm{~d}$ & $12.42 \mathrm{~b}$ & $54.60 \mathrm{c}$ & $12.42 \mathrm{~d}$ & $8.44 \mathrm{c}$ & $7.48 \mathrm{~d}$ & $0.032 \mathrm{~b}$ & 0.022 c & $0.43 \mathrm{~d}$ & $0.39 \mathrm{c}$ & $0.12 \mathrm{~d}$ \\
\hline $\mathrm{F}$ & $156.47 \mathrm{~b}$ & 20.51 a & $119.52 \mathrm{~b}$ & $23.48 \mathrm{~b}$ & $15.44 \mathrm{~b}$ & $12.58 \mathrm{~b}$ & $0.048 \mathrm{a}$ & $0.032 \mathrm{~b}$ & $0.76 \mathrm{~b}$ & $0.68 \mathrm{~b}$ & $0.21 \mathrm{~b}$ \\
\hline B & $143.72 \mathrm{c}$ & 19.49 a & $120.44 \mathrm{~b}$ & 21.53 c & $15.02 \mathrm{~b}$ & $11.44 \mathrm{c}$ & $0.042 \mathrm{a}$ & $0.032 \mathrm{~b}$ & $0.67 \mathrm{c}$ & $0.67 \mathrm{~b}$ & $0.19 \mathrm{c}$ \\
\hline$B+F$ & 165.49 a & 22.47 a & $144.52 \mathrm{a}$ & 30.62 a & $19.61 \mathrm{a}$ & $14.61 \mathrm{a}$ & $0.048 \mathrm{a}$ & $0.041 \mathrm{a}$ & $0.82 \mathrm{a}$ & $0.78 \mathrm{a}$ & $0.25 \mathrm{a}$ \\
\hline $\mathrm{F}$ & ** & ** & $* *$ & ** & ** & ** & ** & ** & $* *$ & $* *$ & ** \\
\hline LSD & 4.85 & 3.57 & 3.89 & 2.63 & 0.45 & 0.50 & 0.01 & 0.008 & 0.014 & 0.014 & 0.012 \\
\hline $\mathrm{CV} \%$ & 5.19 & 5.60 & 9.20 & 6.30 & 1.63 & 2.30 & 12.15 & 14.26 & 1.15 & 1.18 & 3.39 \\
\hline \multicolumn{12}{|c|}{ TS } \\
\hline \multicolumn{12}{|c|}{ Leaf nutrient content $\left(\mathrm{kg} \cdot \mathrm{ha}^{-1}\right)$} \\
\hline Treatment & $\mathrm{N}$ & $\mathrm{P}$ & $\mathrm{K}$ & $\mathrm{Ca}$ & $\mathrm{Mg}$ & S & B & $\mathrm{Cu}$ & $\mathrm{Fe}$ & $\mathrm{Mn}$ & Zn \\
\hline $\mathrm{C}$ & $77.43 \mathrm{~d}$ & 10.61 c & $52.57 \mathrm{~d}$ & 12.49 c & $7.49 \mathrm{~d}$ & $5.56 \mathrm{c}$ & 0.022 c & $0.014 \mathrm{c}$ & $0.37 \mathrm{c}$ & $0.29 \mathrm{~d}$ & $0.12 \mathrm{c}$ \\
\hline $\mathrm{F}$ & $133.45 \mathrm{~b}$ & 18.48 a & $110.81 \mathrm{~b}$ & 24.59 a & $12.66 \mathrm{~b}$ & $12.42 \mathrm{a}$ & $0.038 \mathrm{~b}$ & $0.034 \mathrm{ab}$ & $0.73 \mathrm{a}$ & $0.63 \mathrm{~b}$ & $0.20 \mathrm{a}$ \\
\hline B & 94.47 c & $13.42 \mathrm{~b}$ & 86.37 c & $17.58 \mathrm{~b}$ & 9.67 c & $8.49 \mathrm{~b}$ & $0.034 \mathrm{~b}$ & $0.032 \mathrm{~b}$ & $0.51 \mathrm{~b}$ & $0.46 \mathrm{c}$ & $0.15 \mathrm{~b}$ \\
\hline$B+F$ & $153.68 \mathrm{a}$ & 20.46 a & $140.03 \mathrm{a}$ & 26.54 a & 14.56 a & $12.40 \mathrm{a}$ & $0.048 \mathrm{a}$ & 0.042 a & $0.76 \mathrm{a}$ & 0.73 a & $0.21 \mathrm{a}$ \\
\hline $\mathrm{F}$ & $* *$ & $* *$ & ** & $* *$ & $* *$ & $* *$ & $* *$ & ** & $* *$ & $* *$ & ** \\
\hline LSD & 3.69 & 2.61 & 8.90 & 2.78 & 0.59 & 2.11 & 0.01 & 0.09 & 0.04 & 0.013 & 0.016 \\
\hline CV \% & 1.70 & 8.8 & 4.90 & 7.30 & 2.86 & 11.52 & 14.99 & 15.84 & 3.67 & 1.30 & 5.02 \\
\hline
\end{tabular}

${ }^{* *}$ and ${ }^{*}$ significant at $1 \%$ and 5\%, respectively, by F test. Means followed by the same letter do not differ among themselves, by Tukey Test (P > $0.05)$.

Table 8. Mean productivity of grains of corn in both the systems—second year of management $\left(\mathrm{t} \cdot \mathrm{ha}^{-1}\right)$.

\begin{tabular}{ccc}
\hline Treatment & Maize second year Mean Productivity $\left(\mathbf{t} \cdot \mathbf{h a}^{\mathbf{- 1}}\right)$ \\
Control & AFS & TS \\
Fertilizer & $6.34 \mathrm{bA}$ & $5.76 \mathrm{bB}$ \\
Biomass & $9.43 \mathrm{aA}$ & $8.76 \mathrm{aB}$ \\
Biomass + fertilizer & $9.51 \mathrm{aA}$ & $8.96 \mathrm{aB}$ \\
F & $10.25 \mathrm{aA}$ & $* .94 \mathrm{aB}$ \\
LSD in the columns & $* * * 74$ & 3.71 \\
CV & 0.957 & 0.56 \\
\hline
\end{tabular}

${ }^{* *}$ and ${ }^{*}$ significant at $1 \%$ and $5 \%$, respectively, by F test. Means followed by the same letter do not differ among themselves, by Tukey Test (P > $0.05)$.

Leucaena diversifolia. In TS, treatments $(\mathrm{B}+\mathrm{F})$ and $(\mathrm{F})$ were similar, with significance difference between them and other treatments. There was no significant difference between treatments (F) and (B) in AFS, demonstrating the ability of the tree in the farm system to recycle nutrients and put them at the disposal of cultures.

Foliar analysis and nutrient content of black oat. 
Table 9. Leaf nutrient concentration in corn in AFS and TS—second year of management.

\section{AFS}

\section{Leaf nutrient concentration}

\begin{tabular}{|c|c|c|c|c|c|c|c|c|c|c|c|}
\hline \multicolumn{6}{|c|}{$\mathrm{g} \cdot \mathrm{kg}^{-1}$} & \multicolumn{6}{|c|}{$\mathrm{mg} \cdot \mathrm{kg}^{-1}$} \\
\hline Treatment & $\mathrm{N}$ & $\mathrm{P}$ & K & $\mathrm{Ca}$ & $\mathrm{Mg}$ & S & B & $\mathrm{Cu}$ & $\mathrm{Fe}$ & Mn & $\mathrm{Zn}$ \\
\hline $\mathrm{C}$ & $17.40 \mathrm{c}$ & $2.42 \mathrm{~b}$ & 12.80 & 2.60 & 3.20 & $1.16 \mathrm{~b}$ & $11.00 \mathrm{c}$ & $3.60 \mathrm{~b}$ & 54.40 & $17.80 \mathrm{~b}$ & 24.40 \\
\hline $\mathrm{F}$ & $21.00 \mathrm{~b}$ & $2.60 \mathrm{ab}$ & 14.00 & 3.60 & 3.28 & $1.70 \mathrm{a}$ & $12.00 \mathrm{bc}$ & $5.60 \mathrm{ab}$ & 61.00 & $31.20 \mathrm{ab}$ & 29.00 \\
\hline B & $21.40 \mathrm{ab}$ & $2.70 \mathrm{ab}$ & 14.00 & 3.20 & 3.36 & $1.54 \mathrm{a}$ & $13.80 \mathrm{a}$ & $5.80 \mathrm{ab}$ & 61.20 & $30.40 \mathrm{ab}$ & 24.80 \\
\hline$B+F$ & 24.20 a & $2.78 \mathrm{a}$ & 14.60 & 3.20 & 3.20 & $1.68 \mathrm{a}$ & $13.40 \mathrm{ab}$ & $6.00 \mathrm{a}$ & 61.60 & 36.20 a & 26.00 \\
\hline F & ** & $*$ & NS & NS & NS & ** & ** & * & NS & * & NS \\
\hline LSD & 3.05 & 0.33 & 2.21 & 1.17 & 1.24 & 0.23 & 1.56 & 2.25 & 13.07 & 14.33 & 7.89 \\
\hline CV \% & 7.74 & 6.65 & 8.52 & 19.87 & 18.81 & 8.17 & 6.63 & 22.80 & 11.21 & 26.41 & 16.13 \\
\hline
\end{tabular}

\section{Leaf nutrient concentration}

\begin{tabular}{|c|c|c|c|c|c|c|c|c|c|c|c|}
\hline \multicolumn{7}{|c|}{$\mathrm{g} \cdot \mathrm{kg}^{-1}$} & \multicolumn{5}{|c|}{$\mathrm{mg} \cdot \mathrm{kg}^{-1}$} \\
\hline Treatment & $\mathrm{N}$ & $\mathrm{P}$ & K & $\mathrm{Ca}$ & $\mathrm{Mg}$ & S & B & $\mathrm{Cu}$ & $\mathrm{Fe}$ & Mn & $\mathrm{Zn}$ \\
\hline C & $16.40 \mathrm{c}$ & $1.76 \mathrm{~b}$ & $11.20 \mathrm{~b}$ & 2.60 & 2.80 & $1.70 \mathrm{a}$ & 10.00 & $4.20 \mathrm{~b}$ & 49.00 & $10.80 \mathrm{~b}$ & $20.80 \mathrm{~b}$ \\
\hline F & 19.40 bc & $2.02 \mathrm{~b}$ & $12.40 \mathrm{ab}$ & 2.80 & 2.92 & $1.90 \mathrm{a}$ & 10.40 & $4.40 \mathrm{~b}$ & 50.40 & $17.00 \mathrm{ab}$ & $23.60 \mathrm{ab}$ \\
\hline B & $22.20 \mathrm{ab}$ & $2.08 \mathrm{~b}$ & $13.80 \mathrm{ab}$ & 2.60 & 2.82 & $1.48 \mathrm{~b}$ & 11.00 & $4.80 \mathrm{~b}$ & 57.20 & 18.20 a & $27.60 \mathrm{ab}$ \\
\hline$B+F$ & $24.00 \mathrm{a}$ & $2.60 \mathrm{a}$ & 14.60 a & 2.60 & 3.08 & $1.90 \mathrm{a}$ & 11.60 & $7.40 \mathrm{a}$ & 60.80 & 23.60 a & $30.80 \mathrm{a}$ \\
\hline $\mathrm{F}$ & $* *$ & $* *$ & $*$ & NS & NS & $* *$ & NS & $* *$ & NS & ** & $*$ \\
\hline LSD & 4.12 & 0.35 & 2.74 & 1.03 & 0.48 & 0.30 & 2.09 & 2.19 & 12.07 & 6.78 & 9.01 \\
\hline CV \% & 10.70 & 8.79 & 11.21 & 20.67 & 8.90 & 5.55 & 10.37 & 22.41 & 11.82 & 20.75 & 18.67 \\
\hline
\end{tabular}

** and ${ }^{*}$ significant at $1 \%$ and $5 \%$, respectively, by $\mathrm{F}$ test. Means followed by the same letter do not differ among themselves, by Tukey Test (P > 0.05).

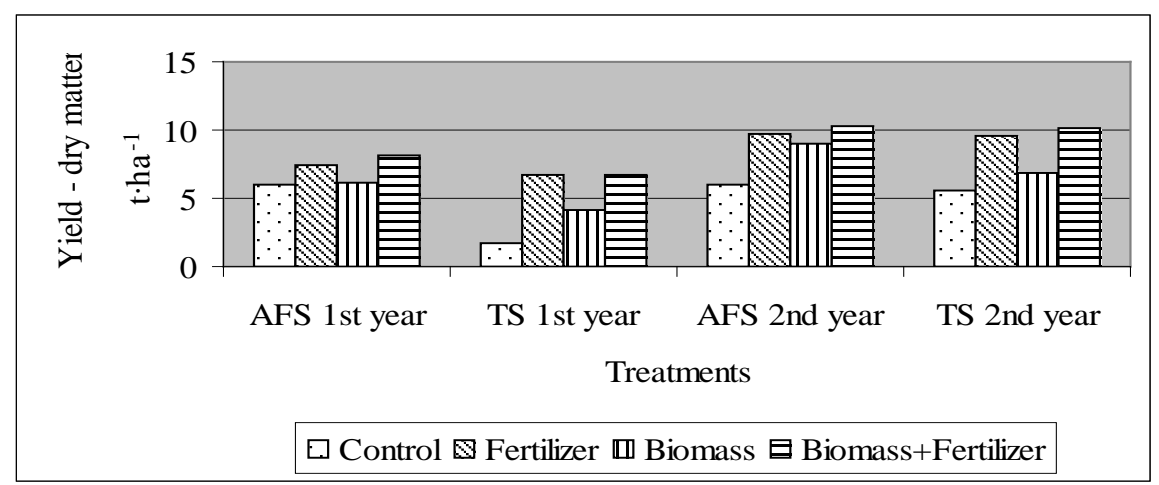

Figure 2. Dry matter yield of black oats. Source: Bertalot (2003) and Bertalot et al. (2010).

The results of the analyses of black oat leaves the first year of management are shown in Table 2. As reported by Camargo et al. (1997) [39], the appropriate levels of nutrients $\left(\mathrm{g} \cdot \mathrm{kg}^{-1}\right.$ ) in the leaves of black oats are as follows: $20-30 \mathrm{~g} \cdot \mathrm{kg}^{-1}$ of N, 2.0 to $5.0 \mathrm{~g} \cdot \mathrm{kg}^{-1} \mathrm{P} ; 15-30 \mathrm{~g} \cdot \mathrm{kg}^{-1} \mathrm{~K} ; 2.5$ to $5.0 \mathrm{~g} \cdot \mathrm{kg}^{-1}$ of Ca, 1.5 to $5.0 \mathrm{~g} \cdot \mathrm{kg}^{-1} \mathrm{of} \mathrm{Mg}$ 
1.5 to $4.8 \mathrm{~g} \cdot \mathrm{kg}^{-1} \mathrm{~S} ; 5-20 \mathrm{mg} \cdot \mathrm{kg}^{-1} \mathrm{~B}$; 5 - $25 \mathrm{mg} \cdot \mathrm{kg}^{-1}$ of $\mathrm{Cu}, 40$ to $150 \mathrm{mg} \cdot \mathrm{kg}^{-1}$ of Fe, 25 to $100 \mathrm{mg} \cdot \mathrm{kg}^{-1}$ of Mn, $0.2-0.3 \mathrm{mg} \cdot \mathrm{kg}^{-1}$ of $\mathrm{Mn}$ and $15-70 \mathrm{mg} \cdot \mathrm{kg}^{-1} \mathrm{Zn}$.

Comparing the values obtained in the AFS, it was observed that only P, Mg and S approached to appropriate levels. In traditional system-TS, only $\mathrm{K}$ approached these levels in treatments (F), (B) and (B + F). In terms of micronutrients, they all showed appropriate levels in both systems.

Using the values of nutrient concentration in the dry matter of black oats and biomass obtained it can be obtained the content of nutrients (Wendt, 1998) [40] and these results are shown in Table 3. It is observed a significant difference in the content of all nutrients in the treatment of both systems, and that in general the nutrients showed higher values in treatments $(B+F)$ and $(F)$ in both systems. Furthermore, with relation to biomass, nutrient values tended to be higher in the AFS.

\section{Maize-first year of management.}

\section{Productivity of maize.}

Productivity of maize in AFS and TS for first year of management is shown in Table 4 and Figure 3.

In the AFS there was not significant difference in productivity between treatments $(B+F),(F)$ and $(B)$, but in relation to treatment $(\mathrm{T})$, showing that an interaction may exist between the biomass of $L$. diversifolia and the presence of trees in this system towards higher yields similar to those obtained with the application of fertilizers, in the first year of management.

In traditional system there was no significant difference between treatments $(B+F)$ and $(F)$, and between treatments (B ) and (F), probably due to nutrients released by the remnants of the culture of oats and biomass of L. diversifolia that favor the production of treatment B.

Table 10. Soil carbon stocked at 0 - $20 \mathrm{~cm}$ soil depth in agroforestry system (AFS) and traditional system (TS), after two years of management.

\begin{tabular}{|c|c|c|}
\hline \multirow[t]{2}{*}{ Trat. } & \multicolumn{2}{|c|}{ Carbon stock at 0 - $20 \mathrm{~cm}$ soil depth $\left(\right.$ Ton $\left.\mathrm{ha}^{-1}\right)$} \\
\hline & AFS & TS \\
\hline $\mathrm{C}$ & $25.45 \mathrm{~b}$ & $23.44 \mathrm{c}$ \\
\hline F & $25.73 \mathrm{~b}$ & $25.73 \mathrm{~b}$ \\
\hline B & 28.39 a & $28.36 \mathrm{a}$ \\
\hline$B+F$ & 28.57 a & $28.56 \mathrm{a}$ \\
\hline F & $* *$ & $* *$ \\
\hline DMS & 0.30 & 0.39 \\
\hline CV \% & 0.52 & 0.78 \\
\hline
\end{tabular}

** and ${ }^{*}$ significant at $1 \%$ and $5 \%$, respectively, by $\mathrm{F}$ test. Lowercase letters in the columns, comparison between treatments for each system. Means followed by the same letter do not differ among themselves, by Tukey Test $(\mathrm{P}>0.05)$.

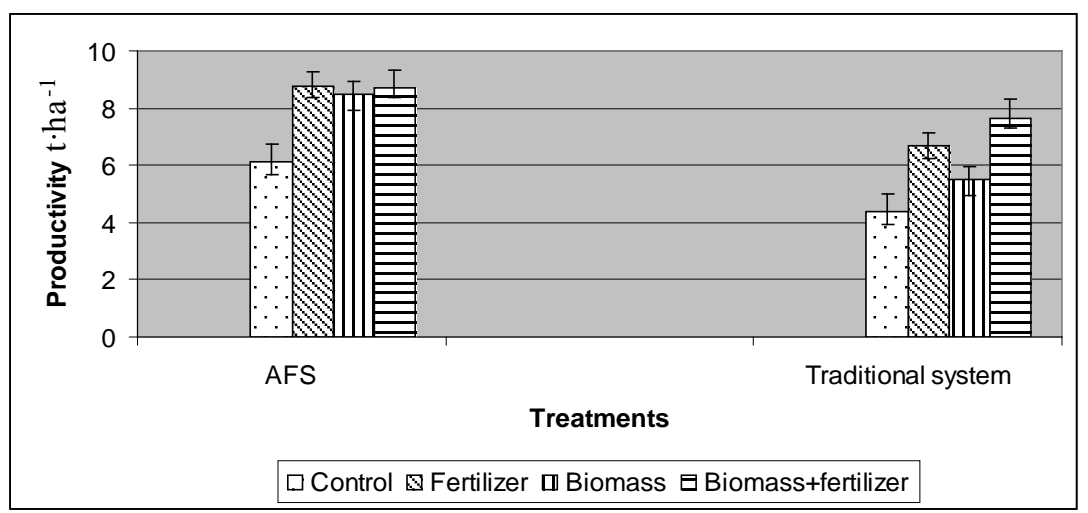

Figure 3. Mean productivity of grains of Maize in function of the treatments, both the systems, first year of management in $\mathrm{t} \cdot \mathrm{ha}^{-1}$. 
Table 11. Temperature data and monthly rainfall during the experiment, from May 2000 to April 2002.

\begin{tabular}{|c|c|c|}
\hline Year & Temp average ${ }^{\circ} \mathrm{C}$ & Rainfall average mm \\
\hline \multicolumn{3}{|l|}{2000} \\
\hline Maio & 18.13 & 10.3 \\
\hline Jun & 18.64 & 12.8 \\
\hline Jul & 15.28 & 55.0 \\
\hline Ago & 17.62 & 73.7 \\
\hline Set & 18.36 & 127.9 \\
\hline Out & 23.24 & 20.3 \\
\hline Nov & 21.42 & 191.9 \\
\hline Dez & 22.28 & 186.8 \\
\hline \multicolumn{3}{|l|}{2001} \\
\hline Jan & 23.57 & 322.2 \\
\hline Fev & 23.86 & 278.2 \\
\hline Mar & 23.29 & 156.6 \\
\hline Abril & 22.64 & 24.9 \\
\hline Maio & 17.56 & 91.0 \\
\hline Jun & 16.69 & 56.9 \\
\hline Jul & 17.35 & 46.1 \\
\hline Ago & 18.04 & 53.8 \\
\hline Set & 18.50 & 87.5 \\
\hline Out & 19.99 & 142.7 \\
\hline Nov & 21.76 & 117.7 \\
\hline Dez & 20.73 & 221.3 \\
\hline \multicolumn{3}{|l|}{2002} \\
\hline Jan & 21.36 & 271.3 \\
\hline $\mathrm{Fev}$ & 20.66 & 209.5 \\
\hline Mar & 23.94 & 150.0 \\
\hline Abril & 22.80 & 2.8 \\
\hline
\end{tabular}

On the system alleys, treatments (F), (B + F) and (B) provided significant increases in grain productivity of $44 \%, 42.29 \%$ and $39.34 \%$ respectively, in relation to treatment $(\mathrm{T})$.

Maize leaf analysis.

The results of the analysis of maize leaf and nutrient concentration in both the systems are shown in Table 5. As Raij and Cantarella (1997) [41] stated, the appropriate nutrient levels in the leaves of maize are the following: 27 - $35 \mathrm{~g} \cdot \mathrm{kg}^{-1} \mathrm{~N} ; 2.0$ - $4.0 \mathrm{~g} \cdot \mathrm{kg}^{-1} \mathrm{P} ; 17$ - $35 \mathrm{~g} \cdot \mathrm{kg}^{-1} \mathrm{~K} ; 2.5$ - $8.0 \mathrm{~g} \cdot \mathrm{kg}^{-1} \mathrm{Ca} ; 1.5$ - $5.0 \mathrm{~g} \cdot \mathrm{kg}^{-1} \mathrm{Mg} ; 1.5$ - $4.8 \mathrm{~g} \cdot \mathrm{kg}^{-1} \mathrm{~S}$; 10 - $25 \mathrm{mg} \cdot \mathrm{kg}^{-1} \mathrm{~B} ; 6$ - $20 \mathrm{mg} \cdot \mathrm{kg}^{-1} \mathrm{Cu} ; 30$ - $250 \mathrm{mg} \cdot \mathrm{kg}^{-1} \mathrm{Fe} ; 20$ - $200 \mathrm{mg} \cdot \mathrm{kg}^{-1} \mathrm{Mn} ; 0.1$ - $0.2 \mathrm{mg} \cdot \mathrm{kg}^{-1}$ Mo e 15 $100 \mathrm{mg} \cdot \mathrm{kg}^{-1} \mathrm{Zn}$.

Comparing the results obtained with the levels considered adequate, it was observed that the $\mathrm{N}$ contents were only suitable in treatment (B + F) of AFS. Values of $\mathrm{N}$ of treatments (F) and (B) tended to approach to ideal values. The TS any treatment showed appropriate levels of $\mathrm{N}$, but treatment $(\mathrm{B}+\mathrm{F})$ tended to approach to ap- 
propriate levels. Due to initial soil chemical characteristics, only the joint application of biomass from L. diversifolia and fertilizers in AFS allowed to achieve the optimal $\mathrm{N}$ in foliar tissue of maize.

It is observed that in AFS there was significant difference between treatments in the concentration of $\mathrm{N}, \mathrm{K}$, $\mathrm{Cu}$, and $\mathrm{Zn}$. Differences in concentrations of N, P, K, Mg, S, B, Cu, Fe, Mn and Zn were observed in TS.

Comparing the results of the foliar tissue analysis in the AFS and TS, it is noted that the numerical values of the concentration of N, P, K, Ca, Mg, S, B, Cu, Fe, Mn, were higher in treatmenst of AFS. This difference may be due to the influence of alley cropping system, but this fact should be further studied.

Bertalot and Mendoza (2006) [42], based on the results of analyzes of leaf tissue samples of Brachiaria decumbens obtained from an alley cropping silvipastoril system with Acacia melanoxylon, in pastures with native trees and sole pastures-without trees, found that nutrient concentrations in B. decumbens grown in AFS were higher than in other systems.

Black oats—second year of management.

The results of the foliar analysis of the different treatments of black oats in the second year of management, for both systems, are shown in Tables 6 and 7.

Comparing these results with nutrients appropriate levels, it was observed that all treatments of agroforestry system are within the range of adequate levels of $\mathrm{P}$; with respect to $\mathrm{Ca}$, just the treatment $(\mathrm{B}+\mathrm{F})$ of the AF system was in accordance to recommended levels; $\mathrm{Mg}$ was adequate in treatments (B + F), (F) and (B) of AFS, probably due to the influence of alleys of $L$. diversifolia and nutrients availability.

It was observed that in the AFS there was a significant difference for $\mathrm{N}, \mathrm{K}, \mathrm{Mg}, \mathrm{S}, \mathrm{Fe}, \mathrm{Mn}, \mathrm{Zn}$, with the highest values for the treatments (B + F) (F) and (B). Concentrations of N, K and Mg were higher in treatments where $L$. diversifolia biomass coverage was present. Regarding micronutrient concentrations of $\mathrm{Mn}$ and $\mathrm{Zn}$ followed the same behavior, not occurring the same fact with the Fe. In the TS, there was a significant difference for K, S, Fe, Mn. For both, macro and micro nutrients, treatments (B + F), (B) and (F) tended to have higher values.

The higher amounts of nutrients in general, were obtained in treatments $(B+F)$ and $(F)$, followed by treatment (B), in both systems, confirming the trend toward performance and yield of biomass dry matter. Greater values where obtained in the AFS system (alley cropping). Regarding these results, Borkert et al. (2003) [43] reported that black oat, although not being a leguminous species, absorbs and recycles reasonable amounts of $\mathrm{N}$, large amounts of $\mathrm{K}, \mathrm{Ca}$ and $\mathrm{Mg}$, and among the micronutrients, $\mathrm{Mn}, \mathrm{Cu}$ and $\mathrm{Zn}$.

\section{Maize-second year of management.}

Maize production presented the second year of cultivation an increasing trend in all treatments in both production systems. This result may be due to the cumulative effect of mineralization and maize straw and oats, along the experiment. How productivity was higher in the AFS system, could also be occurring effect of biological nitrogen fixation, water retention and reduction of extreme microclimate through the rows of $L$. diversifolia.

It can be seen (Table 8 and Figure 4) that was significant difference between treatments and the behavior of these treatments in both systems followed the same pattern. Comparing the two systems, AFS produced significantly more grain maize $\left(\mathrm{t} \cdot \mathrm{ha}^{-1}\right)$ than the TS, considering treatments T, B and F.

There was no significant difference between treatments B + F, B and F, in both systems, but in relation to treatment (T), and the decreasing productivity was B $+\mathrm{F}>\mathrm{B}>\mathrm{F}>\mathrm{C}$ in AFS and $\mathrm{B}>\mathrm{B}+\mathrm{F}>\mathrm{F}>\mathrm{C}$ in TS.

\section{Maize foliar analyses-second year of management.}

The results of the leaf analysis in maize plant are shown in Table 9. The results showed significant differences between treatments in the concentrations of N, P, S, B, Cu, Fe and Mn in agroforestry system, and the concentrations of N, P, K, S, Cu, Fe, Mn and $\mathrm{Zn}$ in the traditional system.

Comparing the AFS and TS, it was observed that the concentration of $\mathrm{N}$ was higher in the AFS treatments, probably due to nitrogen fixation performed through the rows of $L$. diversifolia, the P concentration was higher in treatment (B + F) and (F) of the AFS because plants in AFS suffer less environmental stress (most suitable microclimate, less water stress and temperature. The same behavior was observed in relation to micronutrients, $\mathrm{K}$ concentration was highest in treatment $(\mathrm{B}+\mathrm{F})$ in both systems. Ca concentration was higher in treatment $\mathrm{B}$ of AFS and the concentrations of Mg, B and Fe were higher in all treatments of AFS. Mn concentration was higher in treatment $(\mathrm{B}+\mathrm{A}),(\mathrm{B})$ and $(\mathrm{F})$ of AFS.

\section{Soil Carbon Stock}

Carbon stock reached higher values in treatments B + F and B in both systems with significant difference among these treatments and other two treatments, showing that organic matter production and addition process 


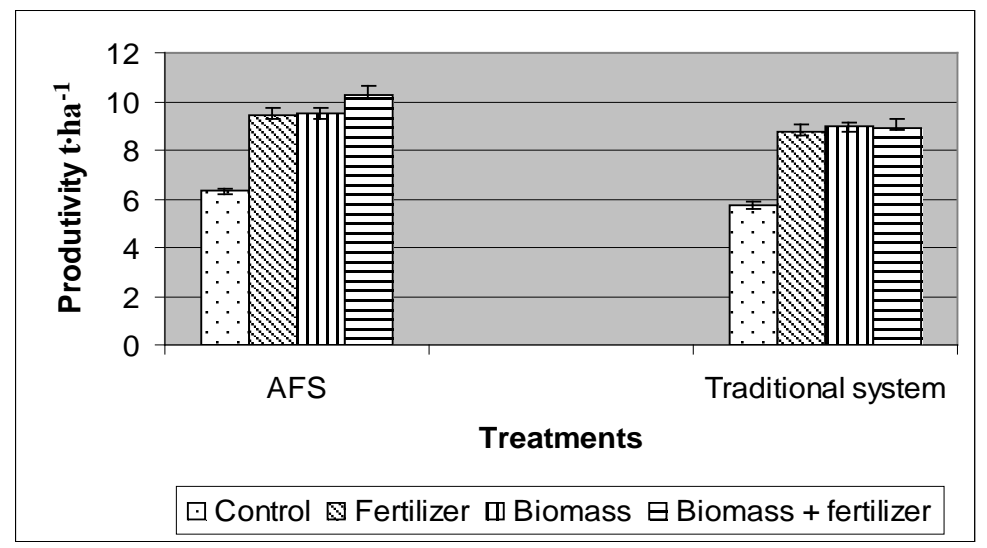

Figure 4. Mean productivity of grains of corn in function of the treatments, both the systems - second year of management in $\mathrm{t} \cdot \mathrm{ha}^{-1}$.

contributes to increase the amount of carbon in the soil profile and it is related to organic matter process of degradation (Table 10).

\section{Conclusion}

The results showed that agroforestry systems contribute to improving naturally cultures productivity, as well as, stimulating increasing concentration of nutrients in leaf tissue. The presence of alley cropping systems and their management practices contribute to soil conservation, as well as for the maintenance of soil fertility and crop productivity. The addition of biomass from prunings of Leucaena diversifolia system in alley systems and successional management with black oats as green manure, associated or not with the addition of chemical fertilizers, helped to increase crop productivity. Traditional agricultural systems are benefited with the adoption of agroecological management techniques of farming. Nitrogen, phosphorus and potassium tended to have higher values in foliar tissue in AFS. It can also be concluded that agricultural production systems that successively in time, handle and/or add biomass allow the accumulation of soil carbon generating benefits for the physical, chemical and biological soil conditioning as well as helping to reduce the presence of carbon in the atmosphere and consequently its impact associated with the greenhouse effect.

\section{References}

[1] Steppler, H.A. and Nair, P.K.R. (1987) Agroforestry: A Decade of Development. International Council for Research in Agroforestry, Nairobi.

[2] Bene, J.G., Beall, H.W. and Cote, A. (1997) Trees, Food and People: Land Management in the Tropics. International Development Research Centre, Ottawa.

[3] Nair, P.K.R. (1984) Soil Productivity Aspects of Agroforestry. International Council for Research in Agroforestry, Nairobi.

[4] Kang, B.T., Reynolds, L. and Atta-Krah, A.N. (1990) Alley Farming. Advances in Agronomy, 43, 315-359. http://dx.doi.org/10.1016/S0065-2113(08)60481-2

[5] Kampen, J. and Budford, J. (1980) Priorities for Alleviating Soil-Related Constraints to Food Crop Production in the Tropics. International Institute of Rural Reconstruction, Manila, 141-145.

[6] Nair, P.K.R. (1985) Classification of Agroforestry Systems. Agroforestry Systems, 3, 97-128. http://dx.doi.org/10.1007/BF00122638

[7] MacDicken, K.G. and Vergara, N.T. (1990) Agroforestry: Classification and Management. John Wiley, New York.

[8] Bertalot, M.J.A., et al. (2004) Retorno de Nutrientes ao solo via Deposição de Serapilheira de Quatro Espécies Leguminosas Arbóreas na Região de Botucatu-SP. Scientia Forestalis, 65, 219-227.

[9] Primavesi, A.M. (1981) Manejo Ecológico do Solo. Nobel, São Paulo, 541p.

[10] Schumacher, M.V., Brun, E.J., Rodrigues, L.M. and dos Santos, E.M. (2003) Retorno de Nutrientes via Deposição de Serapilheira em um Povoamento de Acácia-Negra (Acacia mearnsii De Wild) no Estado do Rio Grande do Sul. Revista Árvore, 27, 791-798. http://dx.doi.org/10.1590/S0100-67622003000600005 
[11] Schumacher, M.V., Brun, E.J., Hernandes, J.I. and König, F.G. (2004) Produção de Serapilheira em uma Floresta de Araucaria angustifolia (Bertol) Kuntze no Município de Pinhal Grande-RS. Revista Árvore, 28, 29-37. http://dx.doi.org/10.1590/S0100-67622004000100005

[12] Bertalot, M.J.A., Guerrini, I.A., Mendoza, E. and Pinto, M.S.V. (2010) Desempenho da Cultura do Milho (Zea mays L.) em Sucessão com Aveia Preta (Avena strigosa Schreb) sob Manejo Agroflorestal e Tradicional. Revista Árvore, 34, 597-608. http://dx.doi.org/10.1590/S0100-6762201000400004

[13] Bertalot, M.J.A., Guerrini, I.A., Mendoza, E. and Pinto, M.S.V. (2008) Análise Econômica da Produção de Milho (Zea mays) sob Sistema Agroflorestal e Tradicional. Revista Ceres, 55, 425-432.

[14] Schmitz, J., Amado, T.J.C., Pedroso, M.T., de A. Acosta, J.A., Spagnollo, E. and Zanon, C. (2012) Adubação Nitrogenada na Aveia Preta: Influência no Rendimento do Milho em Sucessão. http://w3.ufsm.br/ppgcs/congressos/XIV_Reuni\%e3o_Brasileira_Cuiab\%e1/36.pdf

[15] Kaya, B. and Nair, P.R. (2001) Soil Fertility and Crop Yields under Improved-Fallow Systems in Southern Mali. Agroforestry Systems, 52, 1-11. http://dx.doi.org/10.1023/A:1010717629129

[16] Copijn, A.N. (1988) Agrossilvicultura Sustentada por Sistemas Agrícolas Ecologicamente Eficientes. PTA, Rio de Janeiro.

[17] Bertalot, M.J.A. (1997) Crescimento e Avaliação Nutricional de Leguminosas Arbóreas Potenciais Para Ecossistemas Agroflorestais num Solo de Cerrado. MSc. Dissertation, FCA, Unesp, Botucatu-SP.

[18] Bertalot, M.J.A. and Mendoza, E. (1998) Sistemas Agroflorestais. Agricultura Biodinâmica, 80, 22-31.

[19] Haggar, J.P., Tanner, E.V.J., Beer, J.W. and Kass, D.C.L. (1993) Nitrogen Dynamics of Tropical Agroforestry and Annual Cropping Systems. Soil Biology and Biochemistry, 25, 1363-1378. http://dx.doi.org/10.1016/0038-0717(93)90051-C

[20] Marin, A.M.P., Menezes, R.S.C. and Salcedo, I.H. (2007) Productivity of Maize Intercropped or not with Gliricidia Ammended with Two Organic Fertilizers. Pesquisa Agropecuária Brasileira, 42, 669-677. http://www.scielo.br/scielo.php?pid=S0100-204X2007000500009\&script=sci_abstract\&tlng=pt

[21] Marin, A.M.P., Menezes, R.C., Silva, E.D.S. and de S. B. Sampaio, E.V. (2006) Effects of Gliricidia sepium on Soil Nutrients, Microclimate and Maize Yield in an Agroforestry System in Semi-Arid Paraiba. Revista Brasileira de Ciência do Solo, $\mathbf{3 0}$. http://www.scielo.br/scielo.php?pid=S010006832006000300015\&script=sci arttext\&tlng=pt

[22] Siqueira, R. (1999) Sistemas de Preparo em Diferentes Tipos de Coberturas Vegetais de Solo. Doctoral Dissertation, FCA, Unesp, Botucatu-SP.

[23] Sharma, K.L. (2011) Effect of Agroforestry Systems on Soil Quality-Monitoring Assesment. 122-132. http://www.crida.in/DRM1-Winter\%20School/KLS.pdf

[24] Jorge, J.A. (1986) Física e Manejo dos Solos Tropicais. Instituto Campineiro de Ensino Agrícola, Campinas.

[25] Tomé Jr., J.B. (1997) Manual Para Interpretação de Análise de Solo. Livraria e Editora Agropecuária, Guaíba.

[26] Bertalot, M.J.A., Guerrini, I.A. and Mendoza, E. (2002) Growth Parameters and Nutrient Content in Four Multipurpose Tree Species with Potential Characteristics for Agroforestry Systems in a Cerrado Region in Botucatu, São Paulo State, Brazil. Journal of Sustainable Forestry, 15, 87-105. http://dx.doi.org/10.1300/J091v15n02_04

[27] Malavolta, E., Vitti, G.C. and Oliveira, S.A. (1997) Avaliação do Estado Nutricional das Plantas. 2th Edition, Associação Brasileira para Pesquisa da Potassa e do Fosfato, Piracicaba.

[28] Bertalot, M.J.A. (2003) Cultura do Milho (Zea mays L.) em Sucessão com Aveia Preta (Avena strigosa Schreb.) em Áreas sob Manejo Agroflorestal em Aléias com Leucaena diversifolia. Doctoral Dissertation, FCA, Unesp, BotucatuSP.

[29] Leal, A.C., Pavan, M.A., Inoue, M.T. and Koheler, C.W. (1993) Alterações na Fertilidade das Camadas Superficial e Sub-Superficial do Solo por Resíduos de Leucena. 7th Congresso Florestal Brasileiro, Sociedade Brasileira de Silvicultura, Curitiba, 220-222.

[30] Empresa Brasileira De Pesquisa/Agropecuária-EMBRAPA. (1997) Centro Nacional de Pesquisa de Solos, Manual de Métodos de Análise de Solo. 2. Edition, EMBRAPA, Rio de Janeiro.

[31] Raij, B.V. (1983) Avaliação da Fertilidade do Solo. Instituto da Potassa \& Fosfato, Piracicaba.

[32] Raij, B.V., et al. (2001) Análise Química Para Avaliação da Fertilidade de Solos Tropicais. Campinas, Instituto Agronômico de Campinas, Campinas-SP.

[33] Banzatto, D.A. and do N. Kronka, S. (1995) Experimentação Agrícola. Funep, Unesp, Jaboticabal.

[34] Bertalot, M.J.A., Guerrini, I.A., Mendoza Rodriguez, E. and Pinto, M.S.V. (2013) Influence of Agroforestry and Traditional Management of Maize (Zea mays L.) on Soil Fertility. Journal of Sustainable Forestry, 32, 495-511. 
http://dx.doi.org/10.1080/10549811.2013.772084

[35] Luske, B. and van der Kamp, J. (2009) Carbon Sequestration Potential of Reclaimed Desert Soils in Egypt. Louis Bolk Instituut, Driebergen \& Soil and More International, Waddinxveen). Soil \& More International B.V: 100\% Organic Egypt Study—Executive Summary, March, 2012. 7p. http://www.soilandmore.com

[36] Cantarella, H., Quaggio, J.A. and van Raij, B. (2001) Determinação da Matéria Orgânica. In: Análise Química Para Avaliação da Fertilidade de Solos Tropicais, Instituto Agronômico, 173-180.

[37] Derpsch, R. and Calegari, A. (1992) Plantas Para Adubação Verde de Inverno. Londrina, IAPAR, Circular n. 73.

[38] Levien, R. (1999) Condições de Cobertura e Métodos de Preparo do Solo Para a Implantação da Cultura do Milho (Zea mays L.). Doctoral Dissertation, FCA, Unesp, Botucatu-SP.

[39] de O. Camargo, C.E., de Freitas, J.G. and Cantarella, H. (1997) Recomendação de Adubação e Calagem de Aveia Preta Para o Estado de São Paulo. In: Raij, B.V., et al., Eds., Boletim Técnico do Instituto Agronômico, Campinas, 100.

[40] Wendt, V. (1998) Efeito da Adubação Verde de Inverno Associada a Três Doses de NPK, na Cultura do Girassol (Helianthus annus L.) em Dois Sistemas de Semeadura. 122 f. Tese (Doutorado em Agronomia/Agricultura)-Faculdade de Ciências Agronômicas, Universidade Estadual Paulista, Botucatu.

[41] Raij, B.V. and Cantarella, H. (1997) Recomendação de Adubação e Calagem de Milho Para o Estado de São Paulo. In: Raij, B.V., et al., Eds., Boletim Técnico do Instituto Agronômico, Campinas, n. 100, 1-285.

[42] Bertalot, M.J.A., Guerrini, I.A. and Mendoza, E. (2006) Avaliação de Pastagens na Associação Brasileira de Agricultura Biodinâmica. Revista Brasileira de Agroecologia, 1, 1357-1360.

[43] Borkert, C.M., de Almeida Gaudêncio, C., Pereira, J.E., Pereira, L.R. and de Oliveira Junior, A. (2003) Nutrientes Minerais na Biomassa da Parte Aérea em Culturas de Cobertura de Solo. Pesquisa Agropecuária Brasileira, 38, 143-153. http://dx.doi.org/10.1590/S0100-204X2003000100019 